\title{
A VALIDATION OF A PIVOTED POINT ABSORBER TYPE WAVE ENERGY CONVERTER USING CFD
}

\author{
Injun Yang \\ University of Strathclyde \\ Glasgow, Scotland, UK
}

\author{
Tahsin Tezdogan \\ University of Strathclyde \\ Glasgow, Scotland, UK
}

\author{
Atilla Incecik \\ University of Strathclyde \\ Glasgow, Scotland, UK
}

\section{ABSTRACT}

Wave energy is sustainable and clean energy, so it has great potential to be an eco-friendly and lasting renewable energy resource in the future. Recently, a number of researchers have investigated different types of wave energy converters (WECs) using numerical models such as potential theory and Computational Fluid Dynamics (CFD) to enhance the efficiency of such devices. In this paper, a validation of a point absorber type WECs is investigated to capture the movement of the WEC system and to measure the moment on the WEC system. The WEC consists of a lever and a buoy. The geometry is the same as the existing experimental geometry of the reference in order to validate the present numerical simulation. The buoy is connected to the lever and has a hinge on the connection point. Besides, another hinge is installed in the middle of the lever, and the WEC system rotates in the pitch direction. The commercial CFD package Star-CCM+, which solves Reynolds-Averaged Navier-Stokes equations, is employed in this study. In the initial stages of this research, a validation study against published experimental results was conducted. The rotational displacement and the moment on the buoy were compared with the existing experimental data of the reference. The result shows good agreement. In the near future, a study on a new pivoted point absorber WEC device regarding the buoy shape of the WEC device and an operation principle will be performed based on this numerical study.

\section{INTRODUCTION}

The ocean waves are tremendous and enormous resources in the world. It is easy to see that the waves are continuously oscillating and fluctuating from onshore to offshore. There are many types of wave energy converters (WECs) that have been developed as concept designs and a few of the developed WECs installed in a prototype. Developing new concepts of WECs are still an ongoing project. The pioneer of the concept of WEC was Girard [1] who has submitted a patent for the first time. Until now, more than three thousand patents have been registered, and more than 200 WECs were under development in the world in 2017, with different working principle [2]. Some WEC projects have been commenced in order to put them on commercial stage using devices such as different buoy concepts, Oscillating-Water Column (OWC) plants like Pico [3], the Pelamis [4], overtopping
WEC types like the Wave Dragon [5], the point absorber approach used for the SEAREV [6] and the Wave Star [7] device.

The type of WECs can be classified according to their distance to the coast (such as onshore, nearshore, and offshore), their size compared to the wavelength (such as attenuator, terminator and point absorber) and the working principle (such as oscillating water column, oscillating bodies and overtopping) $[8,9]$. It should be noted that those classifications are not sufficiently comprehensive because more concepts are newly introduced. The more extensive knowledge of renewable energy devices can be seen in detail in [10]. Moreover, many concepts of a point absorber type have been introduced depending on the degrees of freedom of a buoy. Those point absorber types can be classified according to whether the WECs have a hinge if only single buoy is considered. According to the presence of hinges, the working principle of a point absorber type WEC can be classified. Some studies provided the examples of point absorber type WEC without hinges, and they considered the only transverse movement of a single buoy and the only heave displacement [11-13]. They have mainly investigated the shape of a buoy in order to enhance the efficiency of power absorption. In addition to the single movement mode, a pivoted point absorber type WEC, which has hinges on the device, has been studied [7, 14-19]. A typical pivoted point absorber type WEC consists of a single buoy, a lever, and a structure to which the lever is connected. The single buoy is connected to the lever and has a hinge on the connection point. Besides, another hinge is installed in the middle of the lever, and the device rotates in the pitch direction. This type is a universal device in the choice of installation sites because it could be installed in onshore, nearshore and offshore as well.

A numerical simulation based on the potential theory has been introduced in the development of WECs, in order to check the performances under wave conditions $[12,15,19,20]$. Recent studies have introduced computational fluid dynamics (CFD) model to evaluate viscous effects action on the buoy and how these influence the performances $[18,21]$. The numerical simulations, both potential theory and CFD model, are a useful tool for the WEC system parameters' analysis and possible future improvement. When the geometry of a buoy and operating principle of WEC is simple, the evaluation showed good agreement with their experimental data. However, if the operating principle of WEC is complicated due to the presence of hinges, the result of numerical simulation was not enough to be satisfied with experimental data. Generally, the method of 
analysing a problem using CFD has been used in a field of marine and ocean engineering for a long time. Many numerical studies have been investigated to improve a numerical towing tank which has the same role as a towing tank in a laboratory including generating waves with a floating object. One of the studies is applying a damping layer, in order to reduce a reflected wave from the outlet of a numerical tank when incoming waves propagate continuously [22]. They have investigated the influence of the thickness of the damping layer and the influence of the parameter of the damping functions via momentum sinks. It provided a good quality of result in order to avoid the reflection wave from the outlet boundary.

The primary objective of this paper is to validate a numerical simulation that has a pivoted point absorber WEC type. An unsteady RANS-based CFD model (Star-CCM+) was used. The wave convergence test according to different mesh sizes has been performed. The damping layer was applied at the end of the computational domain to reduce the reflection wave. Validation study of the pivoted point absorber WEC type in regular waves has been investigated. The moment and motion of the WEC were compared with existing experimental data from $[14,15]$. This numerical study will be a foundation for developing a new pivoted point absorber WEC device installed in a breakwater including the optimisation design of the floater in the future.

\section{NUMERICAL MODEL SETUP}

The governing equations for the simulations are the NavierStokes equations and the continuity equation. An unsteady RANS-based CFD model (Star-CCM+ which is a commercial CFD package, developed by CD-adapco.) was used. It should be noticed that in the RANS solver, the segregated flow model, which solves the flow equation in an uncoupled manner, was applied throughout all simulations in this simulation. By applying a second-order upwind scheme, convection terms in the RANS formulae were discretised. The overall solution procedure was achieved according to a SIMPLE-type algorithm. In order to simulate a WEC behaviour, a Dynamic Fluid Body Interaction (DFBI) model was employed with the WEC system free to move in the pitch and heave directions. The DFBI model allowed the RANS solver to calculate the exciting force and moments acting on the WEC system in order to re-position the rigid body. A realizable $\mathrm{k}-\varepsilon$ two-layer turbulence model was applied with a two-layer all $\mathrm{y}+$ wall treatment model in this study, which has been extensively used for practical application [23]. A second order implicit scheme was utilised for time marching. In order to capture the free surface, a volume of fluid method (VOF) was applied, and overset meshes were used to discretise a computational domain around a pivoted point absorber WEC due to its dynamic motion.

\subsection{WAVE ENERGY CONVERTER SYSTEM}

A pivoted point absorber type WEC in this paper is the same as the reference [15] and illustrated in Fig. 1. This WEC system has two hinges, the first is located on the top of a buoy (a), and the latter is located on a lever (b), which is connected to the buoy.
The device rotates in the pitch direction at the hinge location on the lever, and the hinge ' $b$ ' is located $0.35 \mathrm{~m}$ above the mean water level. The principal dimensions and object characteristics are given in Table 1. Two wave conditions are included for this validation. The wave condition is described in Table 2 . Only mild wave conditions were selected from the existing experimental data to avoid the over-reacted movement of the WEC system. The constant of the damping moment was employed on the lever to match the same condition of the experiment.

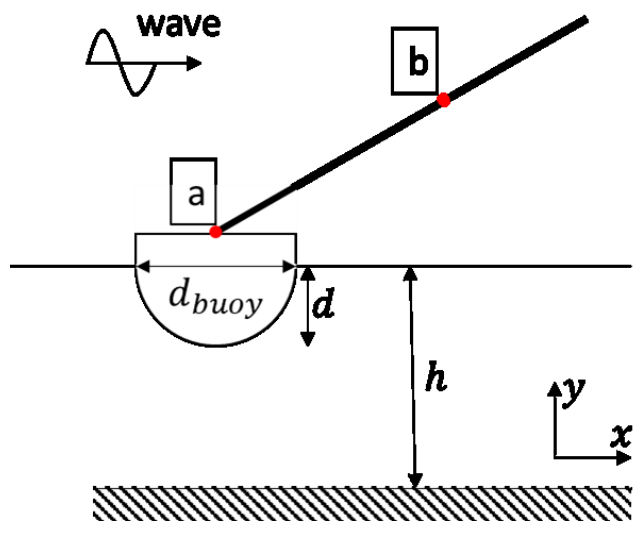

Figure 1 Sketch of the pivoted point absorber WEC with two hinges

Table 1 Numerical value of the WEC model

\begin{tabular}{lll}
\hline Description & Value & Unit \\
\hline \hline Mass of the buoy & 2.972 & $\mathrm{~kg}$ \\
Mass of the lever & 0.851 & $\mathrm{~kg}$ \\
Length of the lever & 0.680 & $\mathrm{~m}$ \\
Diameter of the buoy $\left(\mathrm{d}_{\text {buoy }}\right)$ & 0.254 & $\mathrm{~m}$ \\
Lever arm initial & 0.200 & $\mathrm{~m}$ \\
Moment of Inertia of the buoy & 0.500 & $\mathrm{kgm}^{2}$ \\
Water depth (h) & 0.650 & $\mathrm{~m}$ \\
Draught (d) & 0.104 & $\mathrm{~m}$ \\
PTO damping & $6-15$ & $\mathrm{~N} \mathrm{~m} \mathrm{~s} / \mathrm{rad}$ \\
\hline
\end{tabular}

Table 2 Wave conditions, $\mathrm{H}=$ wave height, $\mathrm{T}=$ wave period, $\mathbf{c}_{\mathbf{c}}=$ damping moment constant on the lever

\begin{tabular}{lcccc}
\hline Waves & $\mathrm{H} / \lambda$ & $\mathrm{H}$ & $\mathrm{T}$ & $\mathrm{c}_{\mathrm{c}}$ \\
& {$[-]$} & {$[\mathrm{m}]$} & {$[\mathrm{sec}]$} & $\begin{array}{c}{[\mathrm{N} \mathrm{m}} \\
\mathrm{s} / \mathrm{rad}]\end{array}$ \\
\hline \hline Regular 1 & 0.055 & 0.09 & 1.0 & 6 \\
Regular 2 & 0.018 & 0.05 & 1.4 & 10
\end{tabular}

\subsection{COMPUTATIONAL DOMAIN}

Fig. 2 illustrates the computational domain with the pivoted point absorber WEC device. The size of the computational domain has been decided by the recommended guideline from 
the references [22, 23]. The numerical domain has six wavelengths $(\lambda)$ long in order to apply the scheme of wave damping properly. The water depth and the width of the numerical tank are $0.65 \mathrm{~m}$ and $1.27 \mathrm{~m}$, respectively. The distance between the inlet of the domain and the WEC device is a wavelength. The damping layer is applied near the outlet of the domain. The thickness of the damping layer is two wavelengths by the recommendation based on [22]. In the damping layer, a combination of linear and quadratic wave damping by Choi and Yoon [24] is implemented in the commercial software Star$\mathrm{CCM}+$. Peric and Abdel-Maksoud [22] has investigated how to determine the damping constant in the equation of linear and quadratic according to the thickness of the damping layer, the wave steepness, and the mesh fineness. The practical recommendation of the damping constant for the linear part $\left(f_{1}\right)$ is

$$
\mathrm{f}_{1}=\Psi_{1} \omega
$$

with $\Psi_{1}=\pi$, wave frequency $\omega$, damping constant for quadratic part $\mathrm{f}_{2}=0$, the thickness of the damping layer $\mathrm{x}_{\mathrm{d}}=$ $2 \lambda$. According to Eq.1, the optimal value of damping constant can be achieved depending on wave conditions.

A fifth-order Stokes wave velocity profile was applied to the inflow and top boundaries. The pressure outlet was set at the down wave boundary, and a symmetry boundary was implemented along the $x-z$ plane to reduce the size of computational resources. Wall boundary condition was placed at the bottom and slip-wall boundary condition was specified at the far sidewall. Fig. 3 illustrates the boundary conditions in the computational domain. These boundary conditions were used as they were reported to give the quickest flow solutions with the high-grade quality for similar simulations carried out utilising Star-CCM + [23]. Applying the velocity inlet boundary condition at the top of the background stops the fluid from sticking to the wall. In other words, it avoids a velocity gradient from occurring between the fluid and the wall, as applying a slip-wall boundary condition. It is of note that the initial flow velocity at all inlet conditions was set to the corresponding velocity of the head waves. Consequently, the flow at the very top of the background is also directed parallel to the outlet boundary. This enables fluid reflections from the top of the domain to be prevented. The top boundary could have been set as a slip-wall or symmetry plane. The selection of boundary conditions from any relevant combination would not affect the flow results significantly. Two independent overset grids (Overset mesh 1 and 2) were applied, in order to consider the movement of the lever and the buoy of the WEC system. The damping moment, which is equivalent to PTO damping in the existing experimental condition, was set on the lever (Fig. 4).

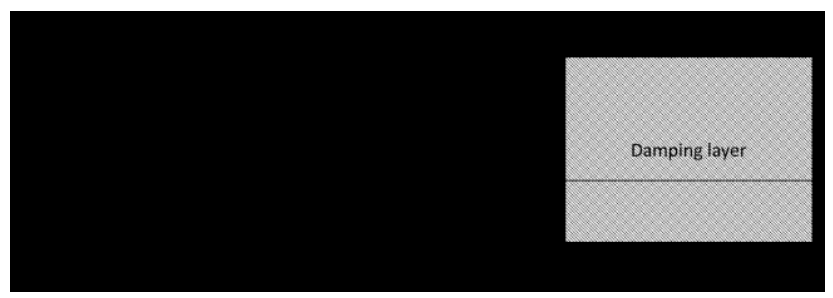

Figure 2 Sketch of the computational domain with the pivoted point absorber WEC device

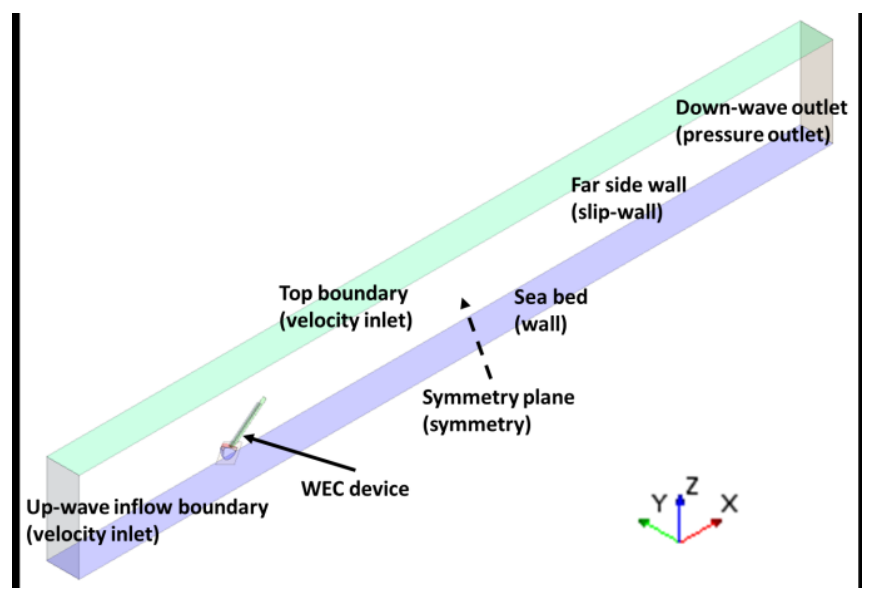

Figure 3 Computational domain boundaries

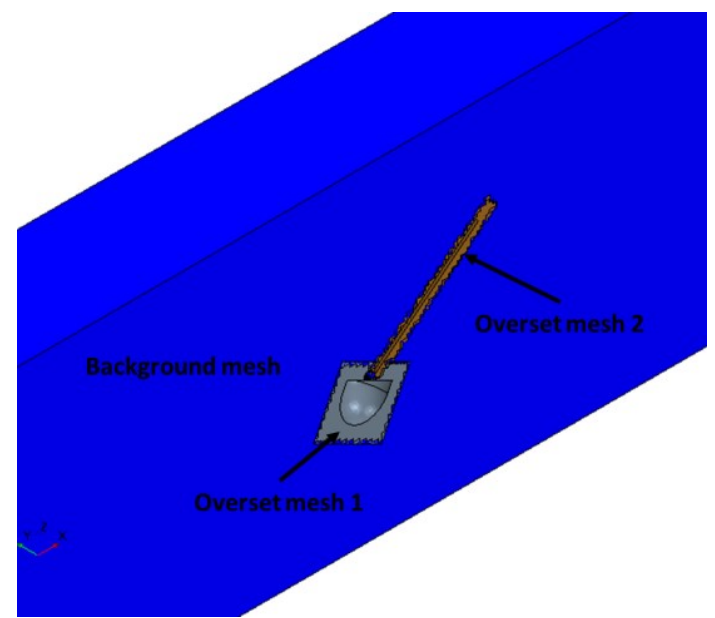

Figure 4 Regions of overset and background mesh

3

NUMERICAL MODELLING OF REGULAR WAVE

In this study, two different grid systems of background mesh and overset mesh were used. The grid size of the background grid was determined by how well the waves were formed. The grid size of $\Delta \mathrm{x}$ (in the wave propagation direction) and $\Delta \mathrm{z}$ (in the vertical direction) were determined by wavelength and wave height, respectively. In order to determine the grid size along the $\mathrm{x}$-direction, four grid systems with different grid sizes were constructed. The wave condition for the comparison is the same as Regular $2(\mathrm{~T}=1.4 \mathrm{~s}, \mathrm{H}=0.05 \mathrm{~m})$. Other variables, such as grid size in the $\mathrm{y}$ and $\mathrm{z}$-direction are equal to $0.064 \mathrm{~m}$ and $\mathrm{H} / 8$, 
respectively, and time-step is equal to $\mathrm{T} / 512$. The peak-to-trough wave elevation $\left(\mathrm{H}_{\mathrm{pt}} / H\right)$ was evaluated to investigate the influence of grid resolution on regular wave simulation. Fig. 5 shows the results of $\mathrm{H}_{\mathrm{pt}} / \mathrm{H}$ according to the number of grids per wavelength. From more than 50 grids per wavelength, $\mathrm{H}_{\mathrm{pt}} / \mathrm{H}$ is showing close to one, which means that the measured wave height is close to the desired wave height. Next, three different grids were generated, which varied according to the number of grids per wave height, and the other parameters of the computational domain are the same. $\Delta \mathrm{x}$ is equal to $\lambda / 100$ for the three different grids. As can be seen in Fig. $5, \mathrm{H}_{\mathrm{pt}} / H$ appears close to one on more than eight grids per wave height. It seemed that a grid resolution of $\Delta \mathrm{x} \approx \lambda / 100$ and $\Delta \mathrm{z}<\mathrm{H} / 8$ is enough for the numerical simulation. Fig. 6 shows the comparison of the measured wave elevation between $\Delta t=$ $T / 512$ and $\Delta t=T / 1024$, in order to confirm the influence of the time-step. The numerical wave probe was located at the same point where the WEC device will be installed. There is no significant difference between the two results, therefore, the time-step of $T / 512$ is enough for the regular wave simulation in this study.

\section{NUMERICAL MODELLING OF A PIVOTED WEC IN WAVES}

In order to compare the result of the moments on the pivoted WEC in regular waves, mesh convergence tests have been conducted, and the influence of the different time-steps has been confirmed. In this study, there are three kinds of grid systems. The Background mesh is for generating regular waves, and the Overset grids 1 and 2 are set to consider the buoy and the lever, respectively (Fig. 7). In the mesh convergence tests, there are three different mesh configuration, and they are listed in Table 3. For the mesh convergence tests, a uniform refinement ratio was chosen to be two, which was applied only to the Overset mesh 1, meaning that the background mesh configuration was not changed. Based on the mesh refinement ratio, the number of cells was determined. The grid size of the Overset mesh 2 to consider the lever does not differ significantly from the grid size of the Background mesh in overlay region and the number of cells of the Overset mesh 2 is set to be constant. The size of Overset mesh 1 has a length of $0.4 \mathrm{~m}$, a width of $0.2 \mathrm{~m}$ and a height of $0.4 \mathrm{~m}$.
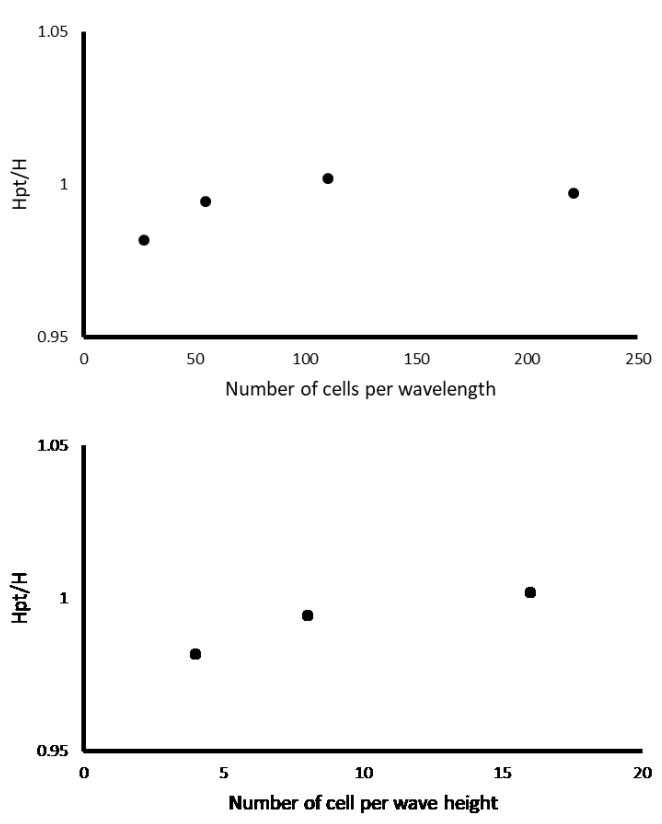

Figure 5 Results of mesh convergence test for regular waves

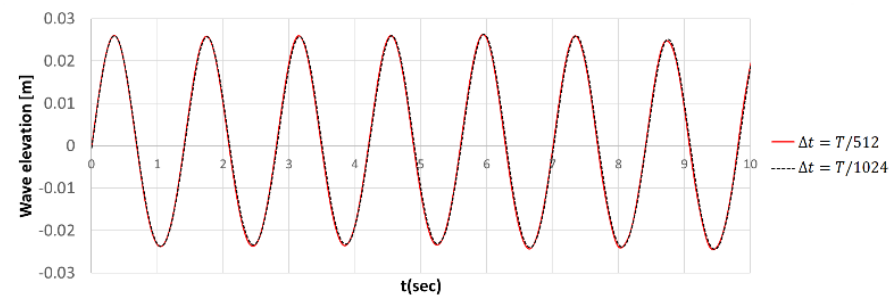

Figure 6 Comparison of the measured wave elevation according to time-step

Table 3 The cell numbers for each mesh configuration as a result of the mesh convergence study with a pivoted WEC

\begin{tabular}{lllll}
\hline \multirow{2}{*}{$\begin{array}{l}\text { Mesh } \\
\text { configuration }\end{array}$} & \begin{tabular}{l} 
Cell number \\
\cline { 2 - 5 } \\
mackground
\end{tabular} & $\begin{array}{l}\text { Overset } \\
\text { mesh 1 }\end{array}$ & $\begin{array}{l}\text { Overset } \\
\text { mesh 2 }\end{array}$ & Total \\
\hline Fine & 354,368 & 201,038 & 21,691 & 577,097 \\
Medium & 354,368 & 51,146 & 21,691 & 427,205 \\
Coarse & 354,368 & 17,574 & 21,691 & 393,633 \\
\hline
\end{tabular}




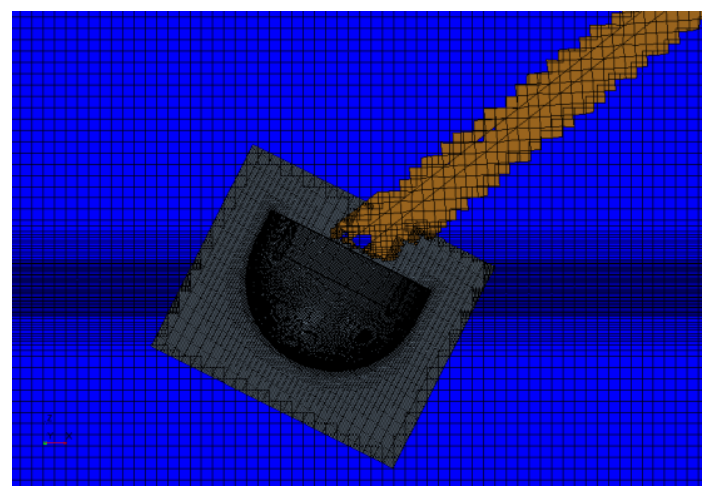

Figure 7 Grid around the WEC device

The grid size of the Background mesh was determined in the previous chapter, and the number of grids of the Background mesh is also fixed. Since the moment on the buoy by the regular waves is the most important problem, the change in the moment due to the grid resolution of the Overset mesh 1 has been confirmed. Fig. 8 shows the variation of the moment on the buoy according to the grid resolution and the comparison to the reference data [15] of experimental and BEM numerical result. The magnitude of the wave excitation forces was obtained by peak-to-peak and trough-to-trough analyses of the steady state for the last six wave periods, which is the same procedure as the reference. The total simulation time is 15 wave periods. The excitation moment magnitude $\left(\left\|M_{e x}\right\|\right)$ is derived by normalising the excitation force by wave amplitude. Here, the experimental result of $\left\|M_{e x}\right\|$ in this study means the average value of the experimental results of the reference. The excitation moment of Fine and Medium grid is close to that of experimental data, as expected. On the other hand, a relatively large discrepancy between the result of Coarse grid and the experimental data was predicted. It is expected that the grid sizes in the overlay region of the Overset mesh 1 and Background mesh could be inappropriate. The grid sizes between the Overset mesh 1 and Background mesh in the overlay region according to the mesh configuration are listed in Table 4. The Background mesh is consist of near free surface region and others region, meaning that the fine meshes are concentrated near the free surface, and the meshes are getting coarser in the z-direction in order to reduce the computational resources. The minimum grid is generated near the buoy of the WEC, the size of the grid is coarser larger towards the overlay region. In the case of Coarse grid, it is confirmed that the grid size of z-direction in Overset mesh 1 is twice as big as the Background mesh. For this reason, interpolation errors caused by the different grids sizes in both sides in the overlay region could be introduced. According to the Star-CCM+ guideline [23], the recommended grids in the overlay region are of a similar size on both the Background and Overset meshes. It is recommended that the influence of the grid size in the overlay region should be investigated as a further study.
Table 4 Comparison of the size of the grid in the overlap region between Overset 1 and Background mesh

\begin{tabular}{|c|c|c|c|c|}
\hline & \multicolumn{3}{|c|}{ The size of the grid } \\
\hline & & $\Delta \mathrm{x}$ & $\Delta y$ & $\Delta \mathrm{z}$ \\
\hline \multirow{3}{*}{ Overset 1} & Fine & 0.008 & 0.032 & 0.002 \\
\hline & Medium & 0.016 & 0.032 & 0.004 \\
\hline & Coarse & 0.016 & 0.032 & 0.008 \\
\hline \multirow[t]{2}{*}{ Background } & $\begin{array}{l}\text { Near free } \\
\text { surface }\end{array}$ & 0.016 & 0.064 & 0.004 \\
\hline & Others & 0.016 & 0.128 & 0.008 \\
\hline
\end{tabular}

Next, comparison of excitation moment amplitude coefficients according to $\Delta t$ has been conducted when the grid system was Medium grid. The time-step convergence test was conducted with three solutions using increased time-steps based on a uniform refinement ration of 2 , starting from $\Delta t=T / 2^{10}$. The result of the comparison of excitation moment amplitude coefficient between the reference data and present numerical data can be seen in Fig. 9. When the $\Delta t$ is the largest in this study, it showed the worst result in comparison to the experimental data, which is even lower than BEM result in the reference. At least, the time-step should be smaller than $T / 2^{9}$ in order to achieve the good quality result of $\left\|M_{e x}\right\|$. Moreover, it is explained that the discrepancy between experimental data and BEM results was due to the absence of high order wave effects in BEM solution. Given this consideration, the first order wave effects and higher order components were considered well in the results of CFD.

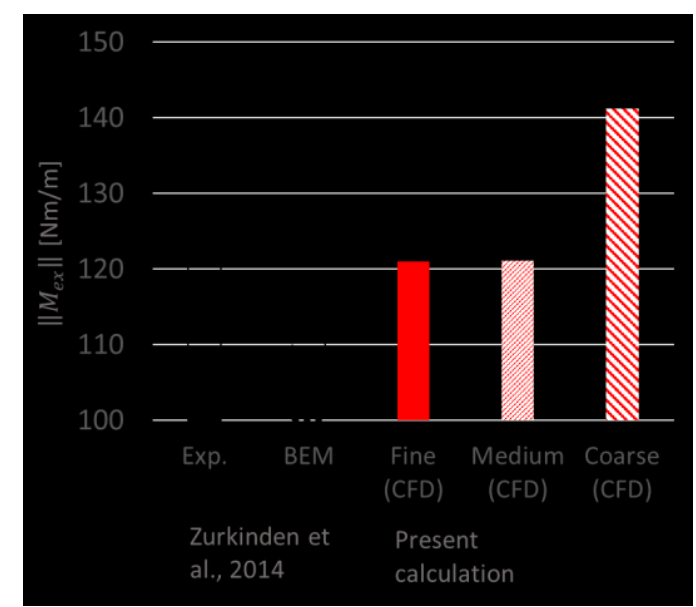

Figure 8 Comparison of excitation moment amplitude coefficients between reference data from Zurkinden et al. (2014) and numerical results according to the number of cells 


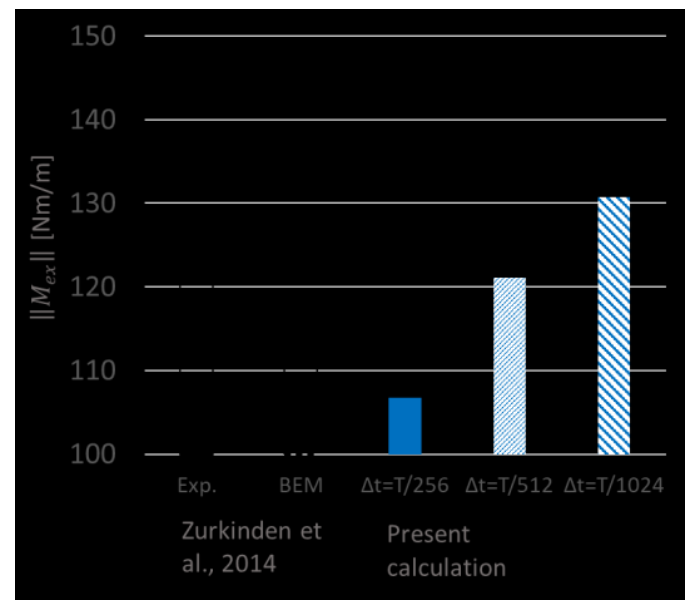

Figure 9 Comparison of excitation moment amplitude coefficients between reference data from Zurkinden et al. (2014) and numerical results according to time-step

\section{COMPARISON OF TIME SIGNAL DATA}

Two different wave conditions were compared with existing experimental data [14]. The first wave with a height of $0.09 \mathrm{~m}$ and a period of 1.0s was given. The second wave with a height of $0.05 \mathrm{~m}$ and a period of $1.4 \mathrm{~s}$ was given. For those two regular waves, the simulation time was around 10 wave periods which shows a repeat of the moment and pitch. In general, experimental data are a good agreement with the numerical results.

Comparison of the rotational displacement between experiment and numerical result is given in Fig. 10. At the trough in the pitch graph, both experimental and numerical data shows good agreement. But at the peak, experimental data shows higher than the numerical result. This is because there was overtopping water on the top of the buoy. Therefore, the presence of the water on the top could affect that the pitch of numerical result is not fully reached. The moment at the hinge on the lever from the numerical simulation and the measured moment by experimental are given in Fig. 10. The trend of the difference between experimental and numerical data is similar to the results of the pitch. Due to the presence of the water on the top, the moment of numerical calculation could be reduced around the peak. Fig. 11 illustrates the comparison of the measured rotational displacement between numerical results and experimental data when the wave has a period of $\mathrm{T}=1.4$ seconds and a height of $0.05 \mathrm{~m}$. Only the rotational displacement can be compared because the time signal data of the moment has not been provided. The compared result of the pitch of the lever shows good agreement with the existing experimental data. Due to the mild wave condition rather than the first wave condition, the recorded pitch by numerical simulation shows good quality and there was no the effect caused by the presence of the water on the top of the buoy.
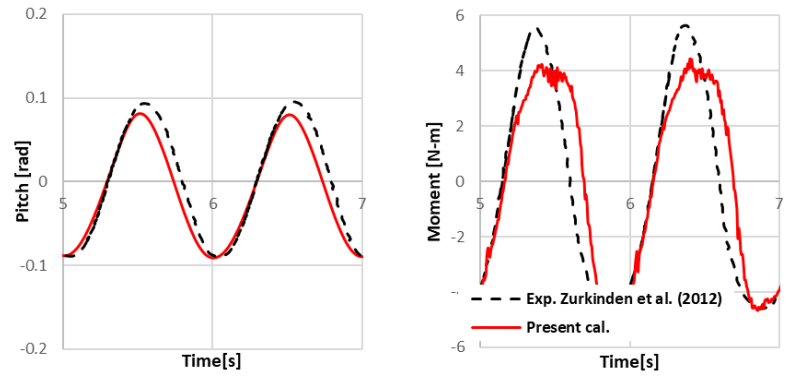

Figure 10 Measured rotational displacement and moment compared with experimental data $[14], T=1.0 s, H=0.09 m$

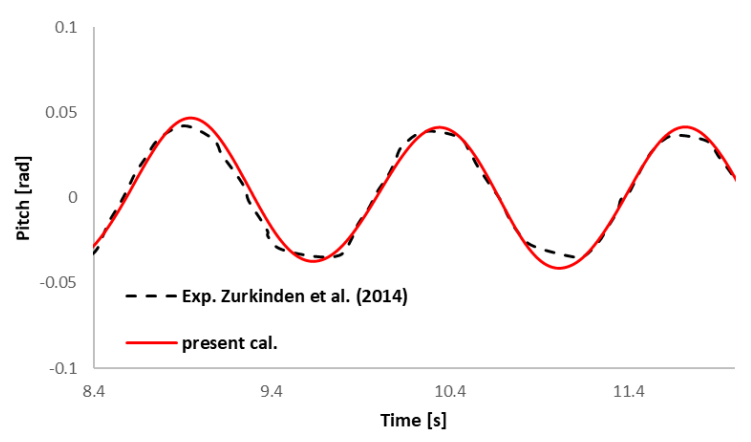

Figure 11 Measured rotational displacement compared with experimental data [15], $\mathrm{T}=1.4 \mathrm{~s}, \mathrm{H}=\mathbf{0 . 0 5 \mathrm { m }}$

\section{CONCLUSIONS}

The main objective of this paper is the validation of the pivoted point absorber WEC device using CFD (Star-CCM+). In order to consider the movement of the WEC device due to the presence of the hinges on both the lever and the top of the buoy, two different overset grids were implemented to both the lever and the buoy. The wave convergence test of the Background mesh, including the scheme of wave damping by applying the damping layer near the down-wave outlet, was performed to determine the number of the grids on the computational domain. Moreover, the time-step converge test of the Background mesh has been conducted.

The grid convergence tests of the Overset mesh for considering the movement of the lever of the WEC device were performed. It is shown that the grid sizes of the Background and Overset mesh in the overlay region is an important factor which should be considered carefully. If the grid size of the Background mesh is bigger than that of the Overset mesh in the overlay region, the numerical results yielded inappropriate value. From the time-step convergence tests of the Overset mesh, the variable of time-step is very sensitive to the result of the moment coefficients. Moreover, the parametric study of the domain size of the Overset mesh is recommended as a further study.

Two different wave conditions were given to evaluate the comparison between the existing experimental data $[14,15]$ and present numerical study. In general, the experimental data of the moment and pitch displacement are good agreement with the 
numerical results. From those comparisons, the possibility of the numerical simulation including the pivoted point absorber WEC device has been confirmed. In the near future, a study on a new pivoted point absorber WEC device regarding the buoy shape of the WEC device and an operation principle will be performed based on this study.

\section{ACKNOWLEDGMENTS}

This study is supported by the University of Strathclyde.

\section{REFERENCES}

[1] D. Ross, Power from the Waves. Oxford University Press, USA, 1995.

[2] A. Babarit, "Wave Energy Conversion Historical Perspective," in Wave Energy Conversion, A. Babarit, Ed.: Elsevier, 2017, pp. 37-98.

[3] F. Neumann, V. Winands, A. Sarmento, L. Energéticas, R. D. L. Ataíde, and S. D. de Rana, "Pico Shoreline OCW: status and new perspectives," Proceedings of the 2nd International Conference on Ocean Energy, ICOE, 2008.

[4] R. Yemm, D. Pizer, C. Retzler, and R. Henderson, "Pelamis: experience from concept to connection," Philos Trans A Math Phys Eng Sci, vol. 370, no. 1959, pp. 365-80, Jan 282012.

[5] J. P. Kofoed, P. Frigaard, E. Friis-Madsen, and H. C. Sørensen, "Prototype testing of the wave energy converter wave dragon," Renewable Energy, vol. 31, no. 2, pp. 181-189, 2006/02/01/ 2006.

[6] M. Ruellan, H. BenAhmed, B. Multon, C. Josset, A. Babarit, and A. Clement, "Design methodology for a SEAREV wave energy converter," IEEE Transactions on Energy Conversion, vol. 25, no. 3, pp. 760-767, 2010.

[7] M. Kramer, L. Marquis, and P. Frigaard, "Performance evaluation of the wavestar prototype," in EWTEC Conference-Proceedings, 2011: Citeseer.

[8] F. d. O. Antonio, "Wave energy utilization: A review of the technologies," Renewable and sustainable energy reviews, vol. 14, no. 3, pp. 899-918, 2010.

[9] A. Pecher and J. P. Kofoed, Handbook of ocean wave energy. Springer, 2017.

[10] A. H. Day et al., "Hydrodynamic modelling of marine renewable energy devices: A state of the art review," $J$ Ocean Engineering, vol. 108, pp. 46-69, 2015.

[11] R. Hager, N. Fernandez, and M. H. Teng, "Experimental study seeking optimal geometry of a heaving body for improved power absorption efficiency," 2012: International Society of Offshore and Polar Engineers.

[12] M. Vantorre, R. Banasiak, and R. Verhoeven, "Modelling of hydraulic performance and wave energy extraction by a point absorber in heave," (in English),
Applied Ocean Research, vol. 26, no. 1-2, pp. 61-72, Feb-Apr 2004.

[13] P. Beirão and C. Malça, Design and analysis of buoy geometries for a wave energy converter. 2013.

[14] A. S. Zurkinden, M. Kramer, M. T. Teimouri, and M. Alves, "Comparison between numerical modeling and experimental testing of a point absorber WEC using linear power take-off system," in ASME 201231 st International Conference on Ocean, Offshore and Arctic Engineering, 2012, pp. 497-506: American Society of Mechanical Engineers.

[15] A. S. Zurkinden, F. Ferri, S. Beatty, J. P. Kofoed, and M. M. Kramer, "Non-linear numerical modeling and experimental testing of a point absorber wave energy converter," (in English), Ocean Engineering, vol. 78, pp. 11-21, Mar 12014.

[16] D. Ionescu and B. Ngwenya, "Innovative design of a sea wave energy harnesser," in Proceedings of the International Conference on Renewable Energies and Power Quality (ICREPQ’14), Cordoba, Spain, 2014, pp. 7-10.

[17] G. Brando, D. P. Coiro, M. Coppola, A. Dannier, A. Del Pizzo, and I. Spina, "Perturb and Observe Control for an Embedded Point Pivoted Absorber," Energies, vol. 9, no. 11, p. 939, 2016.

[18] D. P. Coiro, G. Troise, G. Calise, and N. Bizzarrini, "Wave energy conversion through a point pivoted absorber: Numerical and experimental tests on a scaled model," Renewable Energy, vol. 87, pp. 317-325, 2016. R. Y. Song, M. Q. Zhang, X. H. Qian, X. C. Wang, Y. M. Dai, and J. H. Chen, "A Floating Ocean Energy Conversion Device and Numerical Study on Buoy Shape and Performance," (in English), Journal of Marine Science and Engineering, vol. 4, no. 2, p. 35, Jun 2016.

[20] J. Hardisty, "Experiments with point absorbers for wave energy conversion," (in English), Journal of Marine Engineering and Technology, vol. 11, no. 1, pp. 51-62, Jan 2012.

[21] Y.-H. Yu, Y. J. C. Li, and Fluids, "Reynolds-Averaged Navier-Stokes simulation of the heave performance of a two-body floating-point absorber wave energy system," vol. 73, pp. 104-114, 2013.

[22] R. Perić and M. J. S. T. R. Abdel-Maksoud, "Reliable damping of free-surface waves in numerical simulations," vol. 63, no. 1, pp. 1-13, 2016.

[23] CD-Adapco, "User Guide-STAR-CCM+ Version 13.04," ed: CD-Adapco, 2018.

[24] J. Choi and S. B. Yoon, "Numerical simulations using momentum source wave-maker applied to RANS equation model," Coastal Engineering, vol. 56, no. 10, pp. 1043-1060, 2009. 